EPJ Web of Conferences 41, 04015 (2013)

DOI: $10.1051 /$ epjconf/20134104015

(C) Owned by the authors, published by EDP Sciences, 2013

\title{
Ultra-fast polariton dynamics in an organic microcavity
}

\author{
T. Virgili ${ }^{1}$, D. Coles ${ }^{2}$, A.M. Adawi ${ }^{2+}$, C. Clark $^{3}$, P. Michetti ${ }^{4}$, S.K. Rajendran ${ }^{1}$, D. Brida ${ }^{1}$, D. Polli ${ }^{1}$, \\ G. Cerullo ${ }^{1}$ and D.G. Lidzey ${ }^{2}$
}

${ }^{1}$ IFN, CNR Dipartimento di Fisica, Politecnico di Milano, P.zza Leonardo Da Vinci 32, 20132

Milano, Italy

${ }^{2}$ Department of Physics and Astronomy, University of Sheffield, Hicks Building, Hounsfield Road, Sheffield S3 7RH, United Kingdom

${ }^{3}$ Helia Photonics Ltd. Rosebank Park, Livingston, West Lothian EH54 7EJ, United Kingdom

${ }^{4}$ Institute of Theoretical Physics and Astrophysics, University of Würzburg, D-97074 Würzburg, Germany

${ }^{+}$Present address: Department of Physics University of Hull, Cottingham Road Hull, HU6 7RX, United Kingdom

\begin{abstract}
We study an organic semiconductor microcavity operating in the strongcoupling regime using femtosecond pump-probe spectroscopy. By probing the photoinduced absorption bands, we characterize the time-dependent population densities of states in the two polariton branches. We found evidence of a scattering process from the upper-branch cavity polaritons to the exciton reservoir having a rate of (150 fs $)-1$. A slower process similarly populates lower-branch polaritons with a rate of around (3ps)-1
\end{abstract}

\section{Introduction}

A strongly-coupled microcavity is a structure in which cavity photons and semiconductor excitons undergo coherent coupling, resulting in the emergence of new states termed cavity polaritons [1]. Despite significant interest in inorganic-semiconductor based cavities, the physics of cavities containing organic semiconductors is much less well-understood. At present, relatively little direct experimental validation has been presented for the interplay between exciton-polaritons and 'reservoir' excitons in an organic microcavity. Here, we provide direct evidence of the transfer of population between the exciton reservoir and upper and lower-branch polariton states using femtosecond pump-probe spectroscopy. We show that UP states are able to scatter to the exciton reservoir with a rate of $(150 \mathrm{fs})^{-1}[2]$. We show that our spectroscopy measurements are in good agreement with theoretical models of relaxation processes within strongly-coupled organic semiconductor microcavities, confirming our growing understanding of such photonic systems.

This is an Open Access article distributed under the terms of the Creative Commons Attribution License 2.0, which permits unrestricted use, distribution, and reproduction in any medium, provided the original work is properly cited. 


\section{Experimental details}

The microcavities studied are based on a double dielectric-mirror structure containing a thin-film of a J-aggregated cyanine dye (TDBC). At exciton-photon resonance $\left(\theta=38^{\circ}\right)$ a doublet of cavity polariton states are detected having a Rabi-splitting energy of $120 \mathrm{meV}$ ( see Fig. 1 left panel).

We have performed ultrafast pump-probe spectroscopy on the strongly-coupled microcavity at $45^{\circ}$ angle of incidence. The degenerate pump and probe pulses (delivered at $1 \mathrm{kHz}$ ) were spectrally broad (having a bandwidth of about $380 \mathrm{meV}$ ), peaked at $2.13 \mathrm{eV}$ and had a temporal duration of $15 \mathrm{fs}$. In our setup, the pump and probe pulses had a parallel polarization and were almost collinear. The pump and probe beams were spatially overlapped on the sample and were focused to $\mathrm{a} \approx 80 \mu \mathrm{m}$ diameter spot on the sample surface resulting in an excitation energy density of $\sim 6 \mathrm{~mJ} \mathrm{~cm}^{-2}$. The transmitted probe pulse was then detected via a computer controlled optical multichannel analyzer.
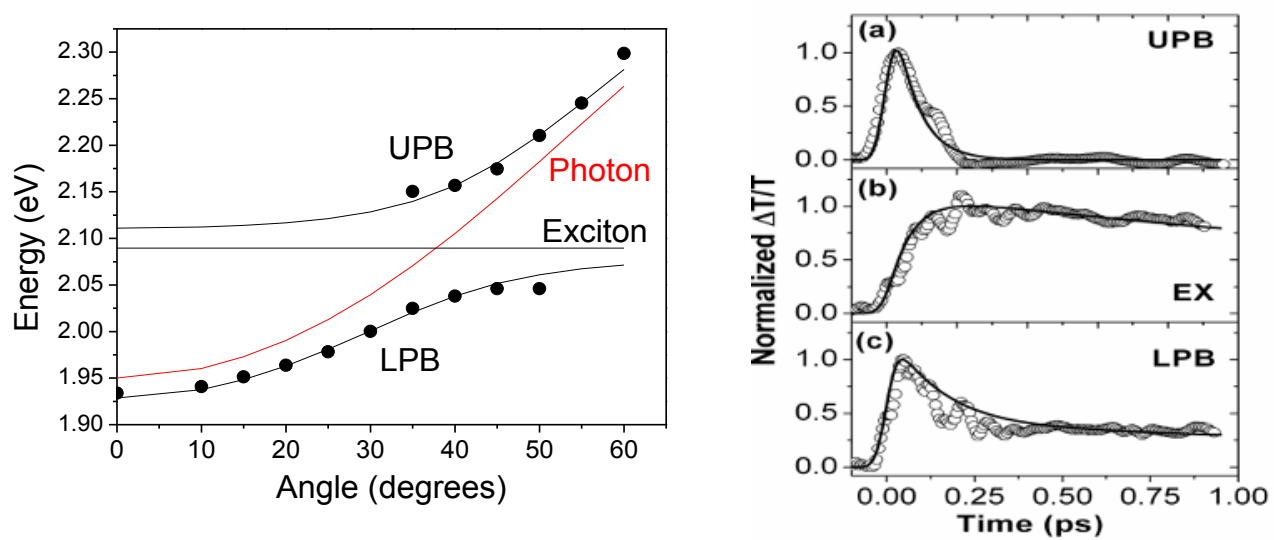

Fig.1. Left panel: The dispersion of the cavity modes as determined from white-light reflectivity measurements (solid circles). The best fit to the measured data points using a two-level model is shown using a solid-line.

Right panel: Parts (a), (b) and (c) show the transient absorption and the best fit (solid line) recorded at energies corresponding to the UP, EX and LP respectively

\section{Results and discussion}

Figure 1 (right panel) shows the transient absorption recorded at $2.03 \mathrm{eV}$ (a), $2.13 \mathrm{eV}$ (b) and $2.17 \mathrm{eV}$ (c) probe energies, mainly corresponding to the population of particles in the upper polariton (UPB), exciton-reservoir (EX) and lower polariton branch (LPB) respectively.

We have modelled the decay dynamics of both polariton branches and the exciton reservoir using the following coupled rate equations:

$$
\begin{aligned}
& \dot{N}_{u}(t)=G_{u}(t)-N_{u}(t)\left[\alpha_{u} \Gamma_{c}+\Gamma_{u \rightarrow x}^{s}\right] \\
& \dot{N}_{x}(t)=G_{x}(t)+N_{u}(t) \Gamma_{u \rightarrow x}^{s}-N_{x}(t) \Gamma_{x \rightarrow l}^{s} \\
& \dot{N}_{l}(t)=G_{l}(t)+N_{x}(t) \Gamma_{x \rightarrow l}^{s}-N_{l}(t) \alpha_{l} \Gamma_{c}
\end{aligned}
$$

Here, $N(t)$ is the time-dependent number of particles, in which the subscripts $u, l$ and $x$ label the UP branch, LP branch and exciton reservoir respectively and $G_{u, l, x}(t)$ represent the time dependent generation terms. The polariton population in the upper and lower branches are then able to undergo optical decay at a rate that is the product of the radiative decay rate of photons from the cavity $\left(\Gamma_{\mathrm{c}}\right)$ 
and the photon-fraction of the polariton state $\left(\alpha_{u, l}\right)$ that at resonance is equal to 0.5. From our twolevel fit to the polariton dispersion curve, we find that $\alpha_{u}=0.54$ and $\alpha_{l}=0.46$ at $\theta=45^{\circ}$. Both excitons and polaritons are also able to undergo scattering to another state (indicated by $\Gamma^{s}$ ). In all cases, the subscripts $u, l$ and $x$ label the initial and final state involved in each decay process. Figure 1 (right panel) shows a best fit (solid line) to the measured pump probe experiments (open circles). As it can be seen, the agreement between the model and the measured data is very good.

\section{Conclusion}

Our model suggests that the radiative lifetime of cavity photons $\left(1 / \Gamma_{c}\right)$ is $\sim 60 \mathrm{fs}$, a value in reasonable agreement with our estimate of 90 fs for cavity with $Q$-factor of approximately 300 . We provide direct evidence that upper-branch cavity-polaritons undergo an ultra-fast scattering process that populates incoherent states in the exciton reservoir with a rate of $(150 \mathrm{fs})^{-1}$ a result in good agreement with theoretical predictions [3-5]. We also find that reservoir excitons scatter into lowerbranch polaritons with a time constant of around $3.2 \mathrm{ps}$.

Understanding such processes could be important in the design and optimization of polariton-based devices in which excitons are initially created following charge-injection or structures in which two semiconductors are strongly coupled to the same optical mode.

\section{References}

[1] M.S. Skolnick, T.A. Fisher and D.M. Whittaker Semicond. Sci. Technol. 13, 645-669 (1998)

[2] T. Virgili, D. Coles, A.M. Adawi, C. Clark P. Michetti, S.K. Rajendran, D. Brida, D. Polli, G. Cerullo and D.G. Lidzey Phys. Rev. B 83, 245309 (2011)

[3] M. Litinskaya, P. Reineker, and V.M. Agranovich, J. Lumin. 119-120, 277-282 (2006)

[4] V. M.Agranovich, and G.C. La Rocca Sol. State Commn. 135, 544-553 (2005).

[5] P. Michetti and G.C. La Rocca Phys. Rev. B 71, 115320-115326 (2005). 